\title{
GENERALIZED $k$-UNIFORMLY CONVEX HARMONIC FUNCTIONS WITH NEGATIVE COEFFICIENTS
}

\author{
SHUHAI LI, HUO TANG, LINA MA AND AO EN
}

\begin{abstract}
In the present paper, we introduce some generalized $k$-uniformly convex harmonic functions with negative coefficients. Sufficient coefficient conditions, distortion bounds, extreme points, Hadamard product and partial sum for functions of these classes are obtained.
\end{abstract}

\section{Introduction and preliminaries}

Let $f_{1}$ and $f_{2}$ be two analytic functions in the open unit disk $U=\{z \in \mathbb{C}:|z|<1\}$. We say that the function $f_{1}$ is subordinate to $f_{2}$ in $U$, and write $f_{1}(z) \prec f_{2}(z)(z \in U)$, if there exists a Schwarz function $\omega$, which is analytic in $U$ with $\omega(0)=0$ and $|\omega(z)|<1 \quad(z \in U)$, such that $f_{1}(z)=f_{2}(\omega(z))(z \in U)$ (see [1]).

A continuous function $f=u+i v$ is a complex valued harmonic function in a complex domain $D$ if both $u$ and $v$ are real harmonic in $D$. In any simply connected domain $D \subset \mathbb{C}$, we can write $f=h+\bar{g}$, where $h$ and $g$ are analytic in $D$. We call $h$ the analytic part and $g$ the co-analytic part of $f$. A necessary and sufficient condition for $f$ to be locally univalent and sense preserving in $D$ is that $\left|h^{\prime}(z)\right|>\left|g^{\prime}(z)\right|$ in $D$ (see [2]; see also [3] - [6]).

Denote by $H$ the class of harmonic functions $f$ that are sense preserving in $U$ and $f$ of the form

$$
f=h+\bar{g}
$$

where

$$
h(z)=z+\sum_{k=2}^{\infty} a_{k} z^{k} \quad \text { and } \quad g(z)=\sum_{k=1}^{\infty} b_{k} z^{k} \quad\left(\left|b_{k}\right|<1\right) .
$$

Received October 8, 2016, accepted January 10, 2017.

2010 Mathematics Subject Classification. 30C45, 30C50, 30C80.

Key words and phrases. Harmonic functions, $k$-uniformly convex, extreme points, distortion bounds, Hadamard product, partial sum.

Corresponding author: Shuhai Li.

Supported by the Natural Science Foundation of China (No. 11561001) and the Natural Science Foundation of Inner Mongolia Province (No. 2014MS0101). 
Let $S_{H}$ denote the family of functions $f=h+\bar{g}$ which harmonic, univalent and sensepreserving in $U$ for which $f(0)=f_{z}(0)-1=0$.

In [2] Clunie and Sheil-Small, investigated the class $S_{H}$ as well as its geometric subclasses and its properties. Since then, there have been several studies related to the class $S_{H}$ and its subclasses. Following Jahangiri [3, 4], Silverman [5], Silverman and Silvia [6], Öztürk et al. [7], and Nagpal and Ravichandran [8] and others have investigated various subclasses of $S_{H}$ and its properties.

Also, we denote by $T_{H}$ the class of harmonic functions $f \in S_{H}$ and

$$
h(z)=z-\sum_{k=2}^{\infty}\left|a_{k}\right| z^{k} \quad \text { and } \quad g(z)=-\sum_{k=1}^{\infty}\left|b_{k}\right| z^{k} \quad\left(\left|b_{k}\right|<1\right) .
$$

Two subclasses of $S$, namely, uniformly convex functions $U C V$ and uniformly starlike functions UST were introduced by Goodman [9], later Rønning [10] and Ma-Minda [11] (see also Rønning [12]) have given more applicable characterization of these classes. Recently, Kanas and Wisniowska [13] (see also [14]) studied class of k-uniformly convex analytic functions.

We using the [15] introduce the following Ma-Minda type function.

Definition 1 (Ma-Minda type function). A function $\varphi(z)$ is said to be Ma-Minda type function if it satisfying the following conditions: $\varphi(z)$ be an analytic function with positive real part in U such that $\varphi(0)=1, \varphi^{\prime}(0)>0$ and $\varphi(z)$ maps $U$ onto a region starlike with respect to 1 and symmetric with respect to the real axis.

Next, we using the Ma-Minda type function, we introduce the following new subclasses of analytic functions.

Definition 2. Let functions $p(z)$ is analytic in $U$ and $p(0)=1$, also let $\varphi(z)$ is Ma-Minda type function. A function $p(z)$ is said to be in the class $U M_{\alpha}(\varphi)$ if it satisfies the following subordination condition

$$
p(z)-\alpha|p(z)-1|<\varphi(z)
$$

where $\alpha \geq 0$.

Let $\mathscr{A}$ denote the class of the functions of the form

$$
f(z)=z+\sum_{k=2}^{\infty} a_{k} z^{k}
$$

which are analytic in $U$.

By making use of the class $U M_{\alpha}(\varphi)$, we introduce the following two harmonic functions. 
Definition 3. Let $A, B \in \mathbb{R},-1 \leq B<A \leq 1, \alpha \geq 0$. A function $f \in S_{H}$ of the form (1.1) is said to be in the class $S_{H}(A, B ; \alpha)$ if and only if

$$
\frac{z f^{\prime}(z)}{z^{\prime} f(z)} \in U M_{\alpha}\left(\frac{1+A z}{1+B z}\right),
$$

where $z^{\prime}=\frac{\partial}{\partial \theta} z$ with $z=r e^{i \theta}, 0 \leq r<1,0 \leq \theta<2 \pi$ and $f^{\prime}(z)=\frac{\partial}{\partial \theta}\left(f\left(r e^{i \theta}\right)\right)$.

Definition 4. Let $A, B \in \mathbb{R}$ and $-1 \leq B<A \leq 1, \alpha \geq 0$. A function $f \in S_{H}$ of the form (1.1) is said to be in the class $K_{H}(A, B ; \alpha)$ if and only if

$$
\frac{\left(z f^{\prime}(z)\right)^{\prime}}{\left(z^{\prime} f(z)\right)^{\prime}} \in U M_{\alpha}\left(\frac{1+A z}{1+B z}\right) .
$$

Clearly, we have

$$
f \in K_{H}(A, B ; \alpha) \Longleftrightarrow z f^{\prime} \in S_{H}(A, B ; \alpha) .
$$

When $\alpha=0$, from Definitions 1-3, we obtain the following subclasses:

$$
\begin{aligned}
& p \in P(A, B) \Longleftrightarrow p(z) \prec \varphi(z), \\
& f \in S_{H}(A, B) \Longleftrightarrow \frac{z f^{\prime}(z)}{z^{\prime} f(z)}<\frac{1+A z}{1+B z}, f \in S_{H}
\end{aligned}
$$

and

$$
f \in K_{H}(A, B) \Longleftrightarrow \frac{\left(z f^{\prime}(z)\right)^{\prime}}{\left(z^{\prime} f(z)\right)^{\prime}} \prec \frac{1+A z}{1+B z}, f \in S_{H} .
$$

Moreover, let us define

$$
\bar{S}_{H}(A, B ; \alpha)=T_{H} \bigcap S_{H}(A, B ; \alpha), \bar{K}_{H}(A, B ; \alpha)=T_{H} \bigcap K_{H}(A, B ; \alpha)
$$

and

$$
\bar{S}_{H}(A, B)=T_{H} \bigcap S_{H}(A, B), \bar{K}_{H}(A, B)=T_{H} \bigcap K_{H}(A, B) .
$$

We further consider the subclasses $S_{H}(A, B ; \alpha)$ and $K_{H}(A, B ; \alpha)$ for $h$ and $g$ given by (1.2). We note that

(1) $S_{H}(1-2 \beta,-1)=S_{H}^{*}(\beta), K_{H}(1-2 \beta,-1)=K_{H}(\beta)(0 \leq \beta<1)$ (see Jahangiri [3, 4]);

(2) $S_{H}(1,-1)=S_{H}^{*}, K_{H}(1,-1)=K_{H}\left(b_{1}=0\right)$ (see Silverman [5] and Silverman and Silvia [6]).

(3) $f \in U C V \Longleftrightarrow 1+\frac{z f^{\prime \prime}(z)}{f^{\prime}(z)} \in U_{1}\left(\frac{1+z}{1-z}\right)\left(b_{k}=0(k \in N) ; f \in \mathscr{A}\right)$ (see [9]-[11]);

(4) $f \in U S V \Longleftrightarrow \frac{z f^{\prime}(z)}{f(z)} \in U_{1}\left(\frac{1+z}{1-z}\right)\left(b_{k}=0(k \in N) ; f \in \mathscr{A}\right)$ (see [10]);

(5) $f \in U C V(\beta) \Longleftrightarrow 1+\frac{z f^{\prime \prime}(z)}{f^{\prime}(z)} \in U_{1}\left(\frac{1+(1-2 \beta) z}{1-z}\right)\left(b_{k}=0(k \in N) ; f \in \mathscr{A}, \beta \in[-1,1)\right)$ and $f \in$ $U S V(\beta) \Longleftrightarrow \frac{z f^{\prime}(z)}{f(z)} \in U_{\alpha}\left(\frac{1+(1-2 \beta) z}{1-z}\right)\left(b_{k}=0(k \in N) ; f \in \mathscr{A}, \alpha \geq 0, \beta \in[-1,1)\right)$ (see [12]); 
(6) $f \in \alpha-U C V \Longleftrightarrow 1+\frac{z f^{\prime \prime}(z)}{f^{\prime}(z)} \in U_{\alpha}\left(\frac{1+z}{1-z}\right)\left(b_{k}=0(k \in N) ; f \in \mathscr{A}, \alpha \geq 0\right)$ and $f \in \alpha-U S V \Longleftrightarrow$ $\frac{z f^{\prime}(z)}{f(z)} \in U_{\alpha}\left(\frac{1+z}{1-z}\right)\left(b_{k}=0(k \in N) ; f \in \mathscr{A}, \alpha \geq 0\right)$ (see [13, 14]).

In this paper, we aim to introduce some new subclasses of harmonic functions defined by subordination and obtain some results including sufficient coefficient conditions, distortion bounds, extreme points, Hadamard product and partial sum for functions of these classes.

\section{Coefficient characterization and distortion theorem}

Lemma 1. Let functions $p(z)$ is analytic in $U$ and $p(0)=1$, also let $\varphi(z)$ is Ma-Minda type function. Then $p(z)$ is said to be in the class $U M_{\alpha}(\varphi)$ if and only if

$$
\left(1-\alpha e^{-i \phi}\right) p(z)+\alpha e^{-i \phi} \prec \varphi(z) \quad(\phi \in \mathbb{R}) .
$$

Proof. Suppose $p(z)-1=|p(z)-1| e^{i \phi}, \phi \in \mathbb{R}$, so we have $|p(z)-1|=(p(z)-1) e^{-i \phi}$. Therefore,

$$
p(z)-\alpha|p(z)-1| \prec \varphi(z) \Longleftrightarrow\left(1-\alpha e^{-i \phi}\right) p(z)+\alpha e^{-i \phi} \prec \varphi(z) \quad(\phi \in \mathbb{R}) .
$$

Remark 1. For $\varphi(z)=\frac{1+A z}{1+B z}(A, B \in \mathbb{R},|B| \leq 1, A \neq B)$, by Lemma 1, we obtain the following result (see [16]):

$$
p-\alpha|p-1|<\frac{1+A z}{1+B z} \Longleftrightarrow\left(1-\alpha e^{-i \phi}\right) p(z)+\alpha e^{-i \phi} \prec \frac{1+A z}{1+B z} \quad(\phi \in \mathbb{R}) .
$$

Using Lemma 1 and (1.5), we get that $f \in S_{H}(A, B ; \alpha)$ if and only if

$$
\left(1-\alpha e^{-i \phi}\right) \frac{z f^{\prime}(z)}{z^{\prime} f(z)}+\alpha e^{-i \phi} \prec \frac{1+A z}{1+B z} \quad(\phi \in \mathbb{R}) .
$$

Also, we get that $f \in K_{H}(A, B ; \alpha)$ if and only if

$$
\left(1-\alpha e^{-i \phi}\right) \frac{\left(z f^{\prime}(z)\right)^{\prime}}{\left(z^{\prime} f(z)\right)^{\prime}}+\alpha e^{-i \phi} \prec \frac{1+A z}{1+B z} \quad(\phi \in \mathbb{R}) .
$$

Theorem 1. Let $f=h+\bar{g}$ be such that $h$ and $g$ are given by (1.2). Also, suppose that $A, B \in \mathbb{R}$ and $-1 \leq B<0<A \leq 1, \alpha \geq 0$. If

$$
\sum_{k=2}^{\infty} \lambda_{k}\left|a_{k}\right|+\sum_{k=1}^{\infty} \mu_{k}\left|b_{k}\right| \leq A-B,
$$

where

$$
k(A-B) \leq \lambda_{k}=(k-1)(1+\alpha-\alpha B)+A-B k(k \geq 2)
$$

and

$$
k(A-B) \leq \mu_{k}=(k+1)(1+\alpha-\alpha B)+A+B k(k \geq 1) .
$$

Then $f(z)$ is sense-preserving harmonic univalent in $U$ and $f \in S_{H}(A, B ; \alpha)$. 
Proof. If $z_{1} \neq z_{2}$,

$$
\begin{aligned}
\left|\frac{f\left(z_{1}\right)-f\left(z_{2}\right)}{h\left(z_{1}\right)-h_{z_{2}}}\right| & \geq 1-\left|\frac{g\left(z_{1}\right)-g\left(z_{2}\right)}{h\left(z_{1}\right)-h_{z_{2}}}\right| \\
& =1-\left|\frac{\sum_{k=1}^{\infty}\left|b_{k}\right|\left(z_{1}^{k}-z_{2}^{k}\right)}{\left(z_{1}-z_{2}\right)+\sum_{k=2}^{\infty}\left|a_{k}\right|\left(z_{1}^{k}-z_{2}^{k}\right)}\right| \\
& >1-\frac{\sum_{k=1}^{\infty} k\left|b_{k}\right|}{1-\sum_{k=2}^{\infty} k\left|a_{k}\right|} \\
& \geq 1-\frac{\sum_{k=1}^{\infty} \frac{\mu_{k}}{A-B}\left|b_{k}\right|}{1-\sum_{k=2}^{\infty} \frac{\lambda_{k}}{A-B}\left|a_{k}\right|} \\
& \geq 0,
\end{aligned}
$$

which proves univalent. Note that $f$ is sense-preserving harmonic in the disc $U$. This is because

$$
\begin{aligned}
\left|h^{\prime}(z)\right| & \geq 1-\sum_{k=1}^{\infty} k\left|a_{k}\right||z|^{k-1}>1-\sum_{k=1}^{\infty} \frac{\lambda_{k}}{A-B}\left|a_{k}\right| \\
& \geq \sum_{k=1}^{\infty} \frac{\mu_{k}}{A-B}\left|b_{k}\right|>\sum_{k=2}^{\infty} k\left|b_{k}\right||z|^{k-1} \geq\left|g^{\prime}(z)\right| .
\end{aligned}
$$

We first show that if the inequality (2.4) holds for the coefficients of $f=h+\bar{g}$, then the required condition (2.2) is satisfied. Using (2.2), we obtain $f \in S_{H}(A, B ; \alpha)$ if and only if there exists an analytic function $\omega(z), \omega(0)=0,|\omega(z)|<1(z \in U)$ such that

$$
\left(1-\alpha e^{-i \phi}\right) \frac{z f^{\prime}(z)}{z^{\prime} f(z)}+\alpha e^{-i \phi}=\frac{1+A \omega(z)}{1+B \omega(z)} \quad(\phi \in \mathbb{R}, z \in U),
$$

or equivalently,

$$
\left|\frac{\left(1-\alpha e^{-i \phi}\right)\left(z f^{\prime}(z)-z^{\prime} f(z)\right)}{\left(A-\alpha e^{-i \phi} B\right) z^{\prime} f(z)-\left(1-\alpha e^{-i \phi}\right) B z f^{\prime}(z)}\right|<1 \quad(\phi \in \mathbb{R}, z \in U),
$$

it suffices to show that

$$
\left|\left(1-\alpha e^{-i \phi}\right)\left(z f^{\prime}(z)-z^{\prime} f(z)\right)\right|-\left|\left(A-\alpha e^{-i \phi} B\right) z^{\prime} f(z)-\left(1-\alpha e^{-i \phi}\right) B z f^{\prime}(z)\right| \leq 0 .
$$

Putting

$$
\gamma_{k, \phi}=(A-k B)+(k-1) B \alpha e^{-i \phi}, \chi_{k, \phi}=(A+k B)+(k+1) B \alpha e^{-i \phi} .
$$

Therefore, from (2.7) we get

$$
\begin{aligned}
& \left|\left(1-\alpha e^{-i \phi}\right)\left(z f^{\prime}(z)-z^{\prime} f(z)\right)\right|-\left|\left(A-\alpha e^{-i \phi} B\right) z^{\prime} f(z)-\left(1-\alpha e^{-i \phi}\right) B z f^{\prime}(z)\right| \\
& \quad=\left|\left(1-\alpha e^{-i \phi}\right)\left[\sum_{k=2}^{\infty}(k-1) a_{k} z^{k}-\sum_{k=1}^{\infty}(k+1) \overline{b_{k} z^{k}}\right]\right|-\left|(A-B) z+\sum_{k=2}^{\infty} \gamma_{k, \phi} a_{k} z^{k}+\sum_{k=1}^{\infty} \chi_{k, \phi} \overline{b_{k} z^{k}}\right|
\end{aligned}
$$




$$
\begin{aligned}
\leq & \sum_{k=2}^{\infty}(1+\alpha)(k-1)\left|a_{k}\right||z|^{k}+\sum_{k=1}^{\infty}(1+\alpha)(k+1)\left|b_{k}\right||z|^{k} \\
& -\left[(A-B)|z|-\sum_{k=2}^{\infty} \gamma_{k, \pi}\left|a_{k}\right||z|^{k}-\sum_{k=1}^{\infty} \chi_{k, \pi}\left|b_{k}\right||z|^{k}\right] \\
= & \sum_{k=2}^{\infty} \lambda_{k}\left|a_{k}\right||z|^{k}+\sum_{k=1}^{\infty} \eta_{k}\left|b_{k}\right||z|^{k}-(A-B)|z| \\
\leq & |z|\left\{\left[\sum_{k=2}^{\infty} \lambda_{k}\left|a_{k}\right||z|^{k-1}+\sum_{k=1}^{\infty} \eta_{k}\left|b_{k}\right||z|^{k-1}\right]-(A-B)\right\} \\
\leq & \sum_{k=2}^{\infty} \lambda_{k}\left|a_{k}\right|+\sum_{k=1}^{\infty} \eta_{k}\left|b_{k}\right|-(A-B) \\
\leq & 0 .
\end{aligned}
$$

By hypothesis the last expression is non-positive. Thus the proof is completed.

Corollary 1. Let $f=h+\bar{g}$ be such that $h$ and $g$ are given by (1.2). Also, suppose that $A, B \in \mathbb{R}$ and $-1 \leq B<0<A \leq 1, \alpha \geq 0$. If

$$
\sum_{k=2}^{\infty} \lambda_{k} k\left|a_{k}\right|+\sum_{k=1}^{\infty} \mu_{k} k\left|b_{k}\right| \leq A-B
$$

then $f(z)$ is sense-preserving harmonic univalent in $U$ and $f \in K_{H}(A, B ; \alpha)$, where $\lambda_{k}$ and $\mu_{k}$ are defined by (2.5) and (2.6), respectively.

Theorem 2. Let $f=h+\bar{g}$ be such that $h$ and $g$ are given by (1.3). Then $f \in \bar{S}_{H}(A, B ; \alpha)$ if and only if the condition (2.4) holds true.

Proof. Since $\bar{S}_{H}(A, B ; \alpha) \subset S_{H}(A, B ; \alpha)$. According to Theorem 1 , we only need to prove the "only if" part of the theorem. Let $f \in \bar{S}_{H}(A, B ; \alpha),-1 \leq B<0<A \leq 1$. Then it satisfies (2.6) or equivalently

$$
\left|\frac{\left(1-\alpha e^{-i \phi}\right)\left[\sum_{k=2}^{\infty}(k-1)\left|a_{k}\right| z^{k}-\sum_{k=1}^{\infty}(k+1)\left|b_{k}\right| \overline{z^{k}}\right]}{(A-B) z-\left[\sum_{k=2}^{\infty} \gamma_{k, \phi}\left|a_{k}\right| z^{k}+\sum_{k=1}^{\infty} \chi_{k, \phi}\left|b_{k}\right| \overline{z^{k}}\right]}\right|<1,
$$

where $\gamma_{k, \phi}$ and $\chi_{k, \phi}$ are defined by (2.8).

From (2.9), we have

$$
\Re\left\{\frac{\left(1-\alpha e^{-i \phi}\right)\left[\sum_{k=2}^{\infty}(k-1)\left|a_{k}\right| z^{k-1}-\sum_{k=1}^{\infty}(k+1)\left|b_{k}\right|\left(\frac{\bar{z}}{z}\right) \overline{z^{k-1}}\right]}{(A-B)-\left[\sum_{k=2}^{\infty}\left[\gamma_{k, \phi}\left|a_{k}\right| z^{k-1}+\sum_{k=1}^{\infty} \chi_{k, \phi}\left|b_{k}\right|\left(\frac{\bar{z}}{z}\right) \overline{z^{k-1}}\right]\right.}\right\}<1,
$$

which is equivalent to

$$
\Re\left\{\rho_{\alpha}(A, B, \phi)\right\}=\Re\left\{1-\frac{\left(1-\alpha e^{-i \phi}\right)\left[\sum_{k=2}^{\infty}(k-1)\left|a_{k}\right| z^{k-1}-\sum_{k=1}^{\infty}(k+1)\left|b_{k}\right|\left(\frac{\bar{z}}{z}\right) \overline{z^{k-1}}\right]}{(A-B)-\left[\sum_{k=2}^{\infty} \gamma_{k, \phi}\left|a_{k}\right| z^{k-1}+\sum_{k=1}^{\infty} \chi_{k, \phi}\left|b_{k}\right|\left(\frac{\bar{z}}{z}\right) \overline{z^{k-1}}\right]}\right\}>0
$$


or

where

$$
\Re\left\{\rho_{\alpha}(A, B, \phi)\right\}=\Re\left\{\frac{(A-B)-F(z)}{(A-B)-G(z)}\right\}>0,
$$

and

$$
\begin{aligned}
F(z)= & {\left[\sum_{k=2}^{\infty} \gamma_{k, \phi}\left|a_{k}\right| z^{k-1}+\sum_{k=1}^{\infty} \chi_{k, \phi}\left|b_{k}\right|\left(\frac{\bar{z}}{z}\right) \overline{z^{k-1}}\right] } \\
& -\left(1-\alpha e^{-i \phi}\right)\left[\sum_{k=2}^{\infty}(k-1)\left|a_{k}\right| z^{k-1}-\sum_{k=1}^{\infty}(k+1)\left|b_{k}\right|\left(\frac{\bar{z}}{z}\right) \overline{z^{k-1}}\right]
\end{aligned}
$$

$$
G(z)=\sum_{k=2}^{\infty} \gamma_{k, \phi}\left|a_{k}\right| z^{k-1}+\sum_{k=1}^{\infty} \chi_{k, \phi}\left|b_{k}\right|\left(\frac{\bar{z}}{z}\right) \overline{z^{k-1}} .
$$

Since $\Re\left\{\rho_{\alpha}(A, B, \phi)\right\}>0$ if and only if there exists a complex-valued function $\omega(z), \omega(0)=$ $0,|\omega(z)|<1(z \in U)$ such that

$$
\rho_{\alpha}(A, B, \phi)=\frac{(A-B)-F(z)}{(A-B)-G(z)}=\frac{1+\omega(z)}{1-\omega(z)},
$$

then (2.11) and (2.12) yield

$$
\begin{aligned}
\Re\left\{\rho_{\alpha}(A, B, \phi)\right\}= & \Re\left\{\frac{1+\omega(z)}{1-\omega(z)}\right\}=\Re\left\{\frac{1-\frac{F(z)}{A-B}}{1-\frac{G(z)}{A-B}}\right\} \\
\geq & \frac{(A-B)-\left[\sum_{k=2}^{\infty}\left|\gamma_{k, \phi}\right|\left|a_{k}\right||z|^{k-1}+\sum_{k=1}^{\infty}\left|\chi_{k, \phi}\right|\left|b_{k}\right||z|^{k-1}\right]}{(A-B)+\sum_{k=2}^{\infty}\left[\left|\gamma_{k, \phi}\right|\left|a_{k}\right||z|^{k-1}+\sum_{k=1}^{\infty}\left|\chi_{k, \phi}\right|\left|b_{k}\right||z|^{k-1}\right]} \\
& -\frac{\left|1-\alpha e^{-i \phi}\right|\left[\sum_{k=2}^{\infty}(k-1)\left|a_{k}\right||z|^{k-1}+(k+1)\left|b_{k}\right||z|^{k-1}\right]}{A-B+\left[\sum_{k=2}^{\infty}\left|\gamma_{k, \phi}\right|\left|a_{k}\right||z|^{k-1}+\sum_{k=1}^{\infty}\left|\chi_{k, \phi}\right|\left|b_{k}\right||z|^{k-1}\right]}>0 .
\end{aligned}
$$

then the above inequality must hold for all $z \in U$. Taking $z=r(0<r<1)$ and $\phi=\pi$, then (2.13) gives

$$
\sum_{k=2}^{\infty} \lambda_{k}\left|a_{k}\right| r^{k-1}+\sum_{k=1}^{\infty} \mu_{k}\left|b_{k}\right| r^{k-1}<A-B .
$$

Letting $r \rightarrow 1^{-}$in (2.14), we will get (2.4).

Corollary 2. Let $f=h+\bar{g}$ be such that $h$ and $g$ are given by (1.3). Also let $\lambda_{k}$ and $\mu_{k}$ be defined by (2.5) and (2.6), respectively. Then $f \in \bar{K}_{H}(A, B ; \alpha)$ if and only if

$$
\sum_{k=2}^{\infty} \lambda_{k} k\left|a_{k}\right|+\sum_{k=1}^{\infty} \mu_{k} k\left|b_{k}\right| \leq A-B .
$$

Theorem 3. Let $f=h+\bar{g} \in T_{H}$ be such that $h$ and $g$ are given by (1.3), $\lambda_{k}$ and $\mu_{k}$ defined by (2.5) and (2.6), respectively. Also, suppose that $\left|b_{1}\right|>\frac{A-B}{\mu_{1}}$ and $\tau_{2}=\min \left\{\lambda_{2}, \mu_{2}\right\}$. If $f \in \bar{S}_{H}(A, B ; \alpha)$, then,

$$
\left(1-\left|b_{1}\right|\right) r-\frac{\mu_{1}\left|b_{1}\right|-(A-B)}{\tau_{2}} r^{2} \leq|f(z)| \leq\left(1+\left|b_{1}\right|\right) r+\frac{\mu_{1}\left|b_{1}\right|-(A-B)}{\tau_{2}} r^{2} .
$$


Proof. Since $f \in \bar{S}_{H}(A, B ; \alpha)$, then by using Theorem 2, we have

$$
\begin{aligned}
|f(z)| & =\left|z-\sum_{k=2}^{\infty}\right| a_{k}\left|z^{k}-\sum_{k=1}^{\infty}\right| b_{k}\left|\overline{z^{k}}\right| \\
& \leq\left(1+\left|b_{1}\right|\right) r+\sum_{k=2}^{\infty}\left|a_{k}\right| r^{2}+\sum_{k=2}^{\infty}\left|b_{k}\right| r^{2} \\
& \leq\left(1+\left|b_{1}\right|\right) r+\frac{A-B}{\tau_{2}} \sum_{k=2}^{\infty}\left(\frac{\tau_{2}}{A-B}\left|a_{k}\right|+\frac{\tau_{2}}{A-B}\left|b_{k}\right|\right) r^{2} \\
& \leq\left(1+\left|b_{1}\right|\right) r+\frac{A-B}{\tau_{2}} \sum_{k=2}^{\infty}\left(\frac{\lambda_{k}}{A-B}\left|a_{k}\right|+\frac{\mu_{k}}{A-B}\left|b_{k}\right|\right) r^{2} \\
& \leq\left(1+\left|b_{1}\right|\right) r+\frac{A-B}{\tau_{2}}\left(\frac{\mu_{1}}{A-B}\left|b_{1}\right|-1\right) r^{2} .
\end{aligned}
$$

and

$$
\begin{aligned}
|f(z)| & \geq\left(1-\left|b_{1}\right|\right) r-\sum_{k=2}^{\infty}\left|a_{k}\right| r^{2}-\sum_{k=2}^{\infty}\left|b_{k}\right| r^{2} \\
& \geq\left(1-\left|b_{1}\right|\right) r-\frac{A-B}{\tau_{2}}\left(\frac{\mu_{1}}{A-B}\left|b_{1}\right|-1\right) r^{2} .
\end{aligned}
$$

The bound (2.16) is sharp for the function given by

$$
f(z)=z \pm\left|b_{1}\right| \bar{z}-\frac{\mu_{1}\left|b_{1}\right|-(A-B)}{\tau_{2}} \bar{z}^{2} .
$$

Using Theorem 3, we obtain the following covering result.

Corollary 3. Let $\left|b_{1}\right|>\frac{A-B}{\mu_{1}}$ and $\tau_{2}=\min \left\{\lambda_{2}, \mu_{2}\right\}$. If $f \in \bar{S}_{H}(A, B ; \alpha)$, then,

$$
\left\{w:|w|<1-\frac{A-B+\left(\mu_{1}-\tau_{2}\right)\left|b_{1}\right|}{\tau_{2}}\right\} .
$$

Corollary 4. Let $f=h+\bar{g}$ be such that $h$ and $g$ are given by (1.3), $\lambda_{k}$ and $\mu_{k}$ defined by (2.5) and (2.6), respectively. Also, suppose that $\left|b_{1}\right|>\frac{A-B}{\mu_{1}}$ and $\tau_{2}=\min \left\{\lambda_{2}, \mu_{2}\right\}$. If $f \in \bar{K}_{H}(A, B ; \alpha)$, then,

$$
\left(1-\left|b_{1}\right|\right) r-\frac{\mu_{1}\left|b_{1}\right|-(A-B)}{2 \tau_{2}} r^{2} \leq|f(z)| \leq\left(1+\left|b_{1}\right|\right) r+\frac{\mu_{1}\left|b_{1}\right|-(A-B)}{2 \tau_{2}} r^{2} .
$$

\section{Extreme points}

Theorem 4. Let $f=h+\bar{g}$ be such that $h$ and $g$ are given by (1.3), $\lambda_{k}$ and $\mu_{k}$ defined by (2.5) and (2.6), respectively. Then $f \in \operatorname{clco} \bar{S}_{H}(A, B ; \alpha)$ if and only if

$$
f(z)=\sum_{k=1}^{\infty}\left[X_{k} h_{k}+Y_{k} g_{k}\right](z \in U),
$$

where 


$$
\begin{aligned}
& h_{1}=z, \\
& h_{k}=z-\frac{A-B}{\lambda_{k}} z^{k} \quad(k \geq 2), \\
& g_{k}=z-\frac{A-B}{\mu_{k}} \bar{z}^{k} \quad(k \geq 1)
\end{aligned}
$$

and

$$
X_{1} \equiv 1-\sum_{k=2}^{\infty} X_{k}-\sum_{k=1}^{\infty} Y_{k}\left(X_{k} \geq 0, Y_{k} \geq 0 ; k=1,2, \ldots\right)
$$

In particular, the extreme points of $f \in \bar{S}_{H}(A, B ; \alpha)$ are $h_{k}$ and $g_{k}$.

Proof. Let $-1 \leq B<0<A \leq 1$, we get

$$
f(z)=\left(\sum_{k=1}^{\infty}\left[X_{k}+Y_{k}\right]\right) z-\sum_{k=2}^{\infty} \frac{A-B}{\lambda_{k}} X_{k} z^{k}-\sum_{k=1}^{\infty} \frac{A-B}{\mu_{k}} Y_{k} \overline{z^{k}}
$$

Since, $0 \leq X_{k} \leq 1 \quad(k=1,2, \ldots)$, we obtain

$$
\sum_{k=2}^{\infty} \frac{\lambda_{k}}{A-B} \frac{A-B}{\lambda_{k}} X_{k} z^{k}+\sum_{k=1}^{\infty} \frac{\mu_{k}}{A-B} \frac{A-B}{\mu_{k}} Y_{k} \overline{z^{k}}=\sum_{k=2}^{\infty} X_{k}+\sum_{k=1}^{\infty} Y_{k}=1-X_{1} \leq 1 .
$$

Consequently, using Theorem 2, we have $f \in \bar{S}_{H}(A, B ; \alpha)$.

Conversely, if $f \in \bar{S}_{H}(A, B ; \alpha)$, then

$$
\left|a_{k}\right| \leq \frac{A-B}{\lambda_{k}}, \quad\left|b_{k}\right| \leq \frac{A-B}{\mu_{k}} .
$$

Putting

$$
X_{k}=\frac{\lambda_{k}\left|a_{k}\right|}{A-B}, \quad Y_{k}=\frac{\mu_{k}\left|b_{k}\right|}{A-B}
$$

and

$$
X_{1}=1-\sum_{k=2}^{\infty} X_{k}-\sum_{k=1}^{\infty} Y_{k} \geq 0
$$

we obtain

$$
\begin{aligned}
f(z) & =z-\sum_{k=2}^{\infty}\left|a_{k}\right| z^{k}-\sum_{k=1}^{\infty}\left|b_{k}\right| \bar{z}^{k} \\
& =\left(\sum_{k=1}^{\infty} X_{k}+\sum_{k=1}^{\infty} Y_{k}\right) z-\sum_{k=2}^{\infty} \frac{A-B}{\lambda_{k}} X_{k} z^{k}-\sum_{k=1}^{\infty} \frac{A-B}{\mu_{k}} Y_{k} \bar{z}^{k} \\
& =\sum_{k=1}^{\infty}\left[h_{k}(z) X_{k}+g_{k}(z) Y_{k}\right] .
\end{aligned}
$$

Thus $f$ can be expressed in the form (3.1). 
Corollary 5. Let $f=h+\bar{g}$ be such that $h$ and $g$ are given by (1.3), $\lambda_{k}$ and $\mu_{k}$ defined by (2.5) and (2.6), respectively. Then $f \in \operatorname{clco} \bar{K}_{H}(A, B ; \alpha)$ if and only if

$$
f(z)=\sum_{k=1}^{\infty}\left[X_{k} h_{k}+Y_{k} g_{k}\right](z \in U)
$$

where

$$
\begin{aligned}
& h_{1}=z, \\
& h_{k}=z-\frac{A-B}{k \lambda_{k}} z^{k} \quad(k \geq 2), \\
& g_{k}=z-\frac{A-B}{k \mu_{k}} \bar{z}^{k} \quad(k \geq 1)
\end{aligned}
$$

and

$$
X_{1} \equiv 1-\sum_{k=2}^{\infty} X_{k}-\sum_{k=1}^{\infty} Y_{k}\left(X_{k} \geq 0, Y_{k} \geq 0\right) \text {. }
$$

In particular, the extreme points of $f \in \bar{K}_{H}(A, B ; \alpha)$ are $h_{k}$ and $g_{k}$.

Theorem 5. The class $\bar{S}_{H}(A, B ; \alpha)$ is closed under convex combinations.

Proof. For $j=1,2, \ldots$, let the functions $f_{j}$ given by

$$
f_{j}(z)=z-\sum_{k=2}^{\infty}\left|a_{j k}\right| z^{k}-\sum_{k=1}^{\infty}\left|b_{j k}\right| \bar{z}^{k}
$$

be in the class $\bar{S}_{H}(A, B ; \alpha)$.

For $0 \leq \eta_{j} \leq 1, \sum_{j=1}^{\infty} \eta_{j}=1$, the convex combinations can be expressed in the form

$$
\sum_{j=1}^{\infty} \eta_{j} f_{j}=z-\sum_{k=2}^{\infty}\left(\sum_{j=1}^{\infty} \eta_{j}\left|a_{j k}\right|\right) z^{k}-\sum_{k=1}^{\infty}\left(\sum_{j=1}^{\infty} \eta_{j}\left|b_{j k}\right|\right) \bar{z}^{k},
$$

then using (2.4), we get

$$
\begin{aligned}
& \sum_{k=2}^{\infty} \frac{\lambda_{k}}{A-B}\left(\sum_{j=1}^{\infty} \eta_{j}\left(\left|a_{j k}\right|\right)\right)+\sum_{k=1}^{\infty} \frac{\mu_{k}}{A-B}\left(\sum_{j=1}^{\infty} \eta_{j}\left(\left|b_{j k}\right|\right)\right) \\
& \quad=\sum_{j=1}^{\infty} \eta_{j}\left\{\sum_{k=2}^{\infty}\left(\frac{\lambda_{k}}{A-B}\left|a_{j k}\right|+\sum_{k=1}^{\infty} \frac{\mu_{k}}{A-B}\left|b_{j k}\right|\right)\right\} \\
& \leq \sum_{j=1}^{\infty} \eta_{j}=1,
\end{aligned}
$$

that is, $\sum_{j=1}^{\infty} \eta_{j} f_{j} \in \bar{S}_{H}(A, B ; \alpha)$.

Corollary 6. The class $\bar{K}_{H}(A, B ; \alpha)$ is closed under convex combinations. 


\section{Hadamard product}

Recently, El-Ashwah and Frasin [17] have studied the Hadamard product of harmonic univalent meromorphic functions. In this section, we establish certain results concerning the Hadamard product of functions belonging to the classes $\bar{K}_{H}(A, B ; \alpha)$ and $\bar{S}_{H}(A, B ; \alpha)$. In order to obtain that, we now introduce a new class of analytic functions.

Definition 5. Let $\delta \geq 0, \alpha \geq 0 ;-1 \leq B<0<A \leq 1$, the function $f=h+\bar{g}$ be such that $h$ and $g$ are given by (1.3), belong to the class $f \in \bar{C}_{H}(A, B ; \alpha, \delta)$ if and only if

$$
\sum_{k=2}^{\infty} k^{\delta} \lambda_{k}\left|a_{k}\right|+\sum_{k=1}^{\infty} k^{\delta} \mu_{k}\left|b_{k}\right| \leq A-B,
$$

where $\lambda_{k}$ and $\mu_{k}$ are defined by (2.5) and (2.6), respectively.

Obviously, for any positive integer $\delta$, we have the following inclusion relation:

$$
\bar{C}_{H}(A, B ; \alpha, \delta) \subset \bar{C}_{H}(A, B ; \alpha, \delta-1) \subset \cdots \subset \bar{C}_{H}(A, B ; \alpha, 2) \subset \bar{K}_{H}(A, B ; \alpha) \subset \bar{S}_{H}(A, B ; \alpha) .
$$

Let the harmonic functions $f_{i}(i=1,2, \ldots, p)$ and $F_{j}(j=1,2, \ldots, q)$ of the form

$$
f_{i}=h_{i}(z)+\overline{g_{i}(z)}=z-\sum_{k=2}^{\infty}\left|a_{k, i}\right| z^{k}-\overline{\sum_{k=1}^{\infty}\left|b_{k, i}\right| z^{k}} \quad\left(\left|b_{k, 1}\right|<1\right)
$$

and

$$
F_{j}=H_{j}(z)+\overline{G_{j}(z)}=z-\sum_{k=2}^{\infty}\left|A_{k, j}\right| z^{k}-\overline{\sum_{k=1}^{\infty}\left|B_{k, j}\right| z^{k}}\left(\left|B_{k, 1}\right|<1\right) .
$$

We define the Hadamard product (or convolution) of $f_{i}$ and $F_{j}$ by

$$
\left(f_{i} * F_{j}\right)(z):=z-\sum_{k=2}^{\infty}\left|a_{k, i}\right|\left|A_{k, j}\right| z^{k}-\overline{\sum_{k=1}^{\infty}\left|b_{k, i}\right|\left|B_{k, j}\right| z^{k}}=:\left(F_{j} * f_{i}\right)(z),
$$

where $i=1,2, \ldots, p$ and $j=1,2, \ldots, q$.

Theorem 6. Let the functions $f_{i}$ defined by (4.2) be in the class $\bar{K}_{H}(A, B ; \alpha)$ for every $i=1,2, \ldots, p$; and let the functions $F_{j}$ defined by (4.3) be in the class $\bar{S}_{H}(A, B ; \alpha)$ for every $j=1,2, \ldots, q$. Then the Hadamard product $f_{1} * f_{2} * \cdots * f_{p} * F_{1} * F_{2} * \cdots * F_{q}(z)$ belongs to the class $\bar{C}_{H}(A, B ; \alpha, 2 p+$ $q-1)$.

Proof. Putting

$$
\xi(z)=f_{1} * f_{2} * \cdots * f_{p} * F_{1} * F_{2} * \cdots * F_{q}(z)
$$

From (4.5) we have

$$
\xi(z)=z-\sum_{k=2}^{\infty}\left(\prod_{i=1}^{p}\left|a_{k, i}\right| \prod_{j=1}^{q}\left|A_{k, i}\right|\right) z^{k}-\overline{\sum_{k=1}^{\infty}\left(\prod_{i=1}^{p}\left|b_{k, i}\right| \prod_{j=1}^{q}\left|B_{k, j}\right|\right) z^{k}} .
$$


To prove the theorem, we need to show that

$$
\sum_{k=2}^{\infty} k^{2 p+q-1} \lambda_{k}\left(\prod_{i=1}^{p}\left|a_{k, i}\right| \prod_{j=1}^{q}\left|A_{k, i}\right|\right)+\sum_{k=1}^{\infty} k^{2 p+q-1} \mu_{k}\left(\prod_{i=1}^{p}\left|b_{k, i}\right| \prod_{j=1}^{q}\left|B_{k, j}\right|\right) \leq A-B,
$$

where $\lambda_{k}$ and $\mu_{k}$ are defined by (2.5) and (2.6), respectively.

Since $f_{i} \in \bar{K}_{H}(A, B ; \alpha)$, we obtain

$$
\sum_{k=2}^{\infty} k \lambda_{k}\left|a_{k, i}\right|+\sum_{k=1}^{\infty} k \mu_{k}\left|b_{k, i}\right| \leq A-B
$$

for every $i=1,2, \ldots, p$. Therefore

$$
k \lambda_{k}\left|a_{k, i}\right| \leq A-B \text { or }\left|a_{k, i}\right| \leq \frac{A-B}{k \lambda_{k}}
$$

and

$$
k \mu_{k}\left|b_{k, i}\right| \leq A-B \text { or }\left|b_{k, i}\right| \leq \frac{A-B}{k \mu_{k}} .
$$

Further, since $\lambda_{k} \geq k(A-B)$ and $\mu_{k} \geq k(A-B)$, we get

$$
\left|a_{k, i}\right| \leq k^{-2} \text { and }\left|b_{k, i}\right| \leq k^{-2} \text {, }
$$

for every $i=1,2, \ldots, p$. Also, since $F_{j} \in \bar{S}_{H}(A, B ; \alpha)$, we have

$$
\sum_{k=2}^{\infty} \lambda_{k}\left|A_{k, j}\right|+\sum_{k=1}^{\infty} \mu_{k}\left|B_{k, j}\right| \leq A-B
$$

for every $j=1,2, \ldots, q$. Hence we obtain

$$
\left|A_{k, j}\right| \leq k^{-1} \text { and }\left|B_{k, j}\right| \leq k^{-1}
$$

for every $j=1,2, \ldots, q$.

Using (4.11) for $i=1,2, \ldots, p$; (4.13) for $j=1,2, \ldots, q-1$ and (4.12) for $j=q$, we obtain

$$
\begin{aligned}
& \sum_{k=2}^{\infty} k^{2 p+q-1} \lambda_{k}\left(\prod_{i=1}^{p}\left|a_{k, i}\right| \prod_{j=1}^{q-1}\left|A_{k, i}\right|\right)\left|A_{k, q}\right|+\sum_{k=1}^{\infty} k^{2 p+q-1} \mu_{k}\left(\prod_{i=1}^{p}\left|b_{k, i}\right| \prod_{j=1}^{q-1}\left|B_{k, j}\right|\right)\left|B_{k, q}\right| \\
& \leq \sum_{k=2}^{\infty} k^{2 p+q-1}\left(\lambda_{k} k^{-2 p} k^{-(q-1)}\right)\left|A_{k, q}\right|+\sum_{k=1}^{\infty} k^{2 p+q-1}\left(\mu_{k} k^{-2 p} k^{-(q-1)}\right)\left|B_{k, q}\right| \\
& =\sum_{k=2}^{\infty} \lambda_{k}\left|A_{k, j}\right|+\sum_{k=1}^{\infty} \mu_{k}\left|B_{k, j}\right| \leq A-B,
\end{aligned}
$$

and therefore $\xi(z) \in \bar{C}_{H}(A, B ; \alpha, 2 p+q-1)$. We note that the required estimate can also be obtained by using (4.11) for $i=1,2, \ldots, p-1$; (4.13) for $j=1,2, \ldots, q$ and (4.8) for $i=p$.

Taking into account the Hadamard product of functions $f_{1} * f_{2} * \cdots * f_{p}$ only, in the proof of Theorem 6, and using (4.11) for $i=1,2, \ldots, p-1$; and relation (4.8) for $i=p$, we are led to 
Corollary 7. Let the functions $f_{i}$ defined by (4.2) be in the class $\bar{K}_{H}(A, B ; \alpha)$ for every $i=1,2, \ldots, p$. Then the Hadamard product $f_{1} * f_{2} * \cdots * f_{p}$ belongs to the class $\bar{C}_{H}(A, B ; \alpha, 2 p-1)$.

Also, taking into account the Hadamard product of functions $F_{1} * F_{2} * \cdots * F_{q}$ only, in the proof of Theorem 6, and using (4.13) for $j=1,2, \ldots, q-1$; and relation (4.12) for $j=q$, we are led to

Corollary 8. Let the functions $F_{j}$ defined by (4.3) be in the class $\bar{S}_{H}(A, B ; \alpha)$ for every $j=$ $1,2, \ldots, q$. Then the Hadamard product $F_{1} * F_{2} * \cdots * F_{q}(z)$ belongs to the class $\bar{C}_{H}(A, B ; \alpha, q-1)$.

\section{Partial sums}

Silverman [18] and Silvia [19] studied partial sum for starlike and convex functions. Recently, Porwal [20], Porwal and Dixit [21] and Porwal [22] have studied analogues interesting results on the partial sum of certain harmonic univalent functions. We consider in this section partial sum of functions in the class $S_{H}(A, B ; \alpha)$ and $K_{H}(A, B ; \alpha)$, and obtain sharp lower bounds for the ratios of real part of $f(z)$ to $f_{m}(z), f(z)$ to $f_{n}(z)$ and $f(z)$ to $f_{m, n}(z)$.

Definition 6. Let the function $f \in S_{H}$ of the form (1.1). Then the sequences of partial sum of functions $f(z)$ are defined by

$$
\begin{gathered}
f_{m}=z+\sum_{k=2}^{m} a_{k} z^{k}+\sum_{k=1}^{\infty} \overline{b_{k} z^{k}}=h_{m}+\bar{g}, \\
f_{n}=z+\sum_{k=2}^{\infty} a_{k} z^{k}+\sum_{k=1}^{n} \overline{b_{k} z^{k}}=h+\overline{g_{n}}
\end{gathered}
$$

and

$$
f_{m, n}=z+\sum_{k=2}^{m} a_{k} z^{k}+\sum_{k=1}^{n} \overline{b_{k} z^{k}}=h_{m}+\overline{g_{n}} .
$$

Theorem 7. Let $f=h+\bar{g}$ be such that $h$ and $g$ are given by (1.3) with $b_{1}=0$, and $f \in \bar{S}_{H}(A, B ; \alpha)$. If $\lambda_{k}$ is defined by (2.5) and

$$
\lambda_{k} \geq \begin{cases}A-B, & k=2,3, \ldots, m, \\ \lambda_{m+1}, & k=m+1, m+2, \ldots, m .\end{cases}
$$

Then

(i) $\Re\left\{\frac{f(z)}{f_{m}(z)}\right\}=\Re\left\{\frac{h+\bar{g}}{h_{m}+\bar{g}}\right\}>1-\frac{A-B}{\lambda_{m+1}}(z \in U, m \in \mathbb{N})$

and

(ii) $\Re\left\{\frac{f_{m}(z)}{f(z)}\right\}=\Re\left\{\frac{h_{m}+\bar{g}}{h+\bar{g}}\right\}>\frac{\lambda_{m+1}}{A-B+\lambda_{m+1}}(z \in U, m \in \mathbb{N})$.

The estimates in (5.4) and (5.5) are sharp for the function given by

$$
f(z)=z+\frac{A-B}{\lambda_{m+1}} \bar{z}^{m+1}(z \in U) .
$$


Proof. (i) Setting

$$
\begin{aligned}
\Psi_{1}(z) & =\frac{\lambda_{m+1}}{A-B}\left\{\frac{f(z)}{f_{m}(z)}-\left(1-\frac{A-B}{\lambda_{m+1}}\right)\right\} \\
& =\frac{\lambda_{m+1}}{A-B}\left\{\frac{h(z)+\overline{g(z)}}{h_{m}(z)+\overline{g(z)}}-\left(1-\frac{A-B}{\lambda_{m+1}}\right)\right\} \\
& =1+\frac{\frac{\lambda_{m+1}}{A-B} \sum_{k=m+1}^{\infty} a_{k} z^{k}}{z+\sum_{k=2}^{m} a_{k} z^{k}+\sum_{k=2}^{\infty} \overline{b_{k} z^{k}}} .
\end{aligned}
$$

In order to get (5.4), it is sufficient to show that $\Re\left(\Psi_{1}(z)\right)>0(z \in U)$, or equivalently

$$
\left|\frac{\Psi_{1}(z)-1}{\Psi_{1}(z)+1}\right| \leq 1 \quad(z \in U)
$$

Since

$$
\left|\frac{\Psi_{1}(z)-1}{\Psi_{1}(z)+1}\right| \leq \frac{\frac{\lambda_{m+1}}{A-B} \sum_{k=m+1}^{\infty}\left|a_{k}\right|}{2-2\left(\sum_{k=2}^{m}\left|a_{k}\right|+\sum_{k=2}^{\infty}\left|b_{k}\right|\right)-\frac{\lambda_{m+1}}{A-B} \sum_{k=m+1}^{\infty}\left|a_{k}\right|},
$$

this last expression is bounded above by 1 , if and only if

$$
\sum_{k=2}^{m}\left|a_{k}\right|+\sum_{k=2}^{\infty}\left|b_{k}\right|+\frac{\lambda_{m+1}}{A-B} \sum_{k=m+1}^{\infty}\left|a_{k}\right| \leq 1 .
$$

It sufficient to show that L. H. S. of (5.9) is bounded above by

$$
\sum_{k=2}^{\infty} \frac{\lambda_{k}}{A-B}\left|a_{k}\right|+\sum_{k=2}^{\infty} \frac{\mu_{k}}{A-B}\left|b_{k}\right|
$$

which is equivalent to

$$
\sum_{k=2}^{m} \frac{\lambda_{k}-(A-B)}{A-B}\left|a_{k}\right|+\sum_{k=2}^{\infty} \frac{\lambda_{k}-(A-B)}{A-B}\left|b_{k}\right|+\sum_{k=m+1}^{\infty} \frac{\lambda_{k}-\lambda_{m+1}}{A-B}\left|a_{k}\right| \geq 0 .
$$

In order to see that the function $f(z)$ of the form (5.6) is extremal, we observe from $z=r e^{\frac{i \pi}{m}}$ that

$$
\frac{f(z)}{f_{m}(z)}=1+\frac{A-B}{\lambda_{m+1}} z^{m} \rightarrow 1-\frac{A-B}{\lambda_{m+1}}\left(r \rightarrow 1^{-}\right) .
$$

(ii) Similarly, if we take

$$
\Psi_{2}(z)=\frac{A-B+\lambda_{m+1}}{A-B}\left\{\frac{f_{m}(z)}{f(z)}-\left(1-\frac{\lambda_{m+1}}{A-B+\lambda_{m+1}}\right)\right\}
$$




$$
=1-\frac{\frac{A-B+\lambda_{m+1}}{A-B}\left(\sum_{k=m+1}^{\infty} a_{k} z^{k}+\sum_{k=2}^{\infty} \overline{b_{k} z^{k}}\right)}{z+\sum_{k=2}^{\infty} a_{k} z^{k}+\sum_{k=2}^{\infty} \overline{b_{k} z^{k}}}
$$

and make use of (5.5), we can deduce that

$$
\left|\frac{\Psi_{2}(z)-1}{\Psi_{2}(z)+1}\right| \leq 1 \quad(z \in U) .
$$

Since

$$
\left|\frac{\Psi_{2}(z)-1}{\Psi_{2}(z)+1}\right| \leq \frac{\frac{A-B+\lambda_{m+1}}{A-B}\left(\sum_{k=m+1}^{\infty}\left|a_{k}\right|+\sum_{k=2}^{\infty} \overline{\left|b_{k}\right|}\right)\left|a_{k}\right|}{2-2\left(\sum_{k=2}^{\infty}\left|a_{k}\right|+\sum_{k=2}^{\infty}\left|b_{k}\right|\right)-\frac{\lambda_{m+1}-A-B}{A-B}\left(\sum_{k=m+1}^{\infty}\left|a_{k}\right|+\sum_{k=2}^{\infty}\left|b_{k}\right|\right)},
$$

this last expression is bounded above by 1 , if and only if

$$
\sum_{k=2}^{m}\left|a_{k}\right|+\sum_{k=2}^{\infty}\left|b_{k}\right|+\frac{\lambda_{m+1}}{A-B} \sum_{k=m+1}^{\infty}\left|a_{k}\right| \leq 1 .
$$

It sufficient to show that L. H. S. of (5.12) is bounded above by

$$
\sum_{k=2}^{\infty} \frac{\lambda_{k}}{A-B}\left|a_{k}\right|+\sum_{k=2}^{\infty} \frac{\mu_{k}}{A-B}\left|b_{k}\right|,
$$

which leads us immediately to the assertion (5.6) of Theorem 7. The bound in (5.5) is sharp for each $m \in \mathbb{N}$ with the extremal function $f(z)$ given by (5.6). Thus we complete the proof of Theorem 7.

Employing the techinques used in Theorem 7, we can prove the following theorems.

Theorem 8. Let $f=h+\bar{g}$ be such that $h$ and $g$ are given by (1.3) with $b_{1}=0$, and $f \in \bar{S}_{H}(A, B ; \alpha)$. If $\mu_{k}$ is defined by (2.5) and

$$
\mu_{k} \geq\left\{\begin{array}{l}
A-B, \quad k=2,3, \ldots, n, \\
\mu_{n+1},
\end{array} \quad k=n+1, n+2, \ldots,\right.
$$

Then

(i) $\Re\left\{\frac{f(z)}{f_{n}(z)}\right\}=\Re\left\{\frac{h+\bar{g}}{h+\bar{g}_{n}}\right\}>1-\frac{A-B}{\mu_{n+1}} \quad(z \in U, n \in \mathbb{N})$

and

(ii) $\Re\left\{\frac{f_{n}(z)}{f(z)}\right\}=\Re\left\{\frac{h+\overline{g_{n}}}{h+\bar{g}}\right\}>\frac{\mu_{n+1}}{A-B+\mu_{n+1}}(z \in U, n \in \mathbb{N})$.

The estimates in (5.13) and (5.14) are sharp for the function given by

$$
f(z)=z+\frac{A-B}{\mu_{n+1}} \bar{z}^{n+1} \quad(z \in U) .
$$


Theorem 9. Let $f=h+\bar{g}$ be such that $h$ and $g$ are given by (1.3) with $b_{1}=0$, and $f \in \bar{S}_{H}(A, B ; \alpha)$. If $\lambda_{k}$ and $\mu_{k}$ are defined by (2.5) and (2.6), respectively, and

$$
\begin{aligned}
& \lambda_{k} \geq \begin{cases}A-B, & k=2,3, \ldots, m, \\
\lambda_{m+1}, & k=m+1, m+2, \ldots,\end{cases} \\
& \mu_{k} \geq \begin{cases}A-B, & k=2,3, \ldots, m, \\
\lambda_{m+1}, & k=m+1, m+2, \ldots .\end{cases}
\end{aligned}
$$

Then

$$
\text { (i) } \Re\left\{\frac{f(z)}{f_{m, n}(z)}\right\}=\Re\left\{\frac{h+\bar{g}}{h_{m}+\overline{g_{n}}}\right\}>1-\frac{A-B}{\lambda_{m+1}}(z \in U, m \in \mathbb{N})
$$

and

$$
\text { (ii) } \Re\left\{\frac{f_{m, n}(z)}{f(z)}\right\}=\Re\left\{\frac{h_{m}+\overline{g_{n}}}{h+\bar{g}}\right\}>\frac{\lambda_{m+1}}{A-B+\lambda_{m+1}}(z \in U, m \in \mathbb{N}) \text {. }
$$

The estimates in (5.16) and (5.17) are sharp for the function given by (5.6).

Proof. (i) Setting

$$
\begin{aligned}
\Psi_{3}(z) & =\frac{\lambda_{m+1}}{A-B}\left\{\frac{f(z)}{f_{m, n}(z)}-\left(1-\frac{A-B}{\lambda_{m+1}}\right)\right\} \\
& =\frac{\lambda_{m+1}}{A-B}\left\{\frac{h(z)+\overline{g(z)}}{h_{m}(z)+\overline{g_{n}(z)}}-\left(1-\frac{A-B}{\lambda_{m+1}}\right)\right\} \\
& =1+\frac{\frac{\lambda_{m+1}}{A-B}\left(\sum_{k=m+1}^{\infty} a_{k} z^{k}+\sum_{k=n+1}^{\infty} \overline{b_{k} z^{k}}\right)}{z+\sum_{k=2}^{m} a_{k} z^{k}+\sum_{k=2}^{n} \overline{b_{k} z^{k}}} .
\end{aligned}
$$

In order to obtain (5.16), it is sufficient to show that $\Re\left(\Psi_{3}(z)\right)>0(z \in U)$, or equivalently

$$
\left|\frac{\Psi_{3}(z)-1}{\Psi_{3}(z)+1}\right| \leq 1 \quad(z \in U) .
$$

Since

$$
\left|\frac{\Psi_{3}(z)-1}{\Psi_{3}(z)+1}\right| \leq \frac{\frac{\lambda_{m+1}}{A-B}\left(\sum_{k=m+1}^{\infty}\left|a_{k}\right|+\sum_{k=n+1}^{\infty}\left|b_{k}\right|\right)}{2-2\left(\sum_{k=2}^{m}\left|a_{k}\right|+\sum_{k=2}^{n}\left|b_{k}\right|\right)-\frac{\lambda_{m+1}}{A-B}\left(\sum_{k=m+1}^{\infty}\left|a_{k}\right|+\sum_{k=n+1}^{\infty}\left|b_{k}\right|\right)},
$$

this last expression is bounded above by 1 , if and only if

$$
\sum_{k=2}^{m}\left|a_{k}\right|+\sum_{k=2}^{\infty}\left|b_{k}\right|+\frac{\lambda_{m+1}}{A-B}\left(\sum_{k=m+1}^{\infty}\left|a_{k}\right|+\sum_{k=n+1}^{\infty}\left|b_{k}\right|\right) \leq 1 .
$$

It sufficient to show that L. H. S. of (5.18) is bounded above by

$$
\sum_{k=2}^{\infty} \frac{\lambda_{k}}{A-B}\left|a_{k}\right|+\sum_{k=2}^{\infty} \frac{\mu_{k}}{A-B}\left|b_{k}\right|,
$$


which is equivalent to

$$
\sum_{k=2}^{m} \frac{\lambda_{k}-(A-B)}{A-B}\left|a_{k}\right|+\sum_{k=2}^{\infty} \frac{\mu_{k}-(A-B)}{A-B}\left|b_{k}\right|+\sum_{k=m+1}^{\infty} \frac{\lambda_{k}-\lambda_{m+1}}{A-B}\left|a_{k}\right|+\sum_{k=n+1}^{\infty} \frac{\mu_{k}-\lambda_{m+1}}{A-B}\left|b_{k}\right| \geq 0,
$$

which readily yields the assertion (5.18) of Theorem ??. Similarly, we easily get the assertion (5.19) of Theorem ??. The estimates in (5.16) and (5.17) are sharp for the function given by (5.6).

Theorem 10. Let $f=h+\bar{g}$ be such that $h$ and $g$ are given by (1.3) with $b_{1}=0$, and $f \in$ $\bar{S}_{H}(A, B ; \alpha)$. If $\lambda_{k}$ and $\mu_{k}$ are defined by (2.5) and (2.6), respectively, and

$$
\begin{aligned}
& \lambda_{k} \geq \begin{cases}A-B, & k=2,3, \ldots, n, \\
\mu_{n+1}, & k=n+1, n+2, \ldots,\end{cases} \\
& \mu_{k} \geq \begin{cases}A-B, & k=2,3, \ldots, n, \\
\mu_{n+1}, & k=n+1, n+2, \ldots,\end{cases}
\end{aligned}
$$

then

and

$$
\text { (i) } \Re\left\{\frac{f(z)}{f_{m, n}(z)}\right\}=\Re\left\{\frac{h+\bar{g}}{h_{m}+\overline{g_{n}}}\right\}>1-\frac{A-B}{\mu_{n+1}}(z \in U, n \in \mathbb{N})
$$

$$
\text { (ii) } \Re\left\{\frac{f_{m, n}(z)}{f(z)}\right\}=\Re\left\{\frac{h_{m}+\overline{g_{n}}}{h+\bar{g}}\right\}>\frac{\mu_{n+1}}{A-B+\mu_{n+1}}(z \in U, n \in \mathbb{N}) \text {. }
$$

The estimates in (5.20) and (5.21) are sharp for the function given by (5.6) and (5.15), respectively.

Remark 2. By specializing the parameters $A, B$ and $\alpha$, we obtain the corresponding results for various subclasses mentioned in the introduction.

\section{Acknowledgements}

The present investigation was partly supported by the Natural Science Foundation of People's Republic of China under Grant 11561001 and the Natural Science Foundation of Inner Mongolia of People's Republic of China under Grant 2014MS0101. Also, the authors would like to thank the referees of the paper for their helpful suggestions.

\section{References}

[1] P. L. Duren. Univalent Functions. Grundlehren der Mathematischen Wissenschaften, Band 259, SpringerVerlag, New York, Berlin, Heidelberg and Tokyo, 1983.

[2] J. Clunie and T. Sheil Small. Harmonic univalent functions, Ann. Acad. Sci. Fenn. Ser. A I Math., 39 (1984), $3-25$. 
[3] J. M. Jahangiri, Coefficient bounds and univalent criteria for harmonic functions with negative coefficients, Ann. Univ. Marie-Curie Sklodowska Sect. A, 52 (1998), 57-66.

[4] J. M. Jahangiri, Harmonic functions starlike in the unit disc, J. Math. Anal. Appl., 235 (1999), 470-477.

[5] H. Silverman, Harmonic univalent function with negative coefficients, J. Math. Anal. Appl., 220 (1998), 283289.

[6] H. Silverman and E. M. Silvia, Subclasses of harmonic univalent functions, New Zealand J. Math., 28 (1999), 275-284.

[7] M. Öztürk, S. Yalcin and M. Yamankaradeniz, Convex subclass of harmonic starlike functions, Appl. Math. Comput., 154 (2004), 449-459.

[8] S. Nagpal and V. Ravichandran, A comprehensive class of harmonic functions defined by convolution and its connection with integral transforms and hypergeometric functions, Stud. Univ. Babes-Bolyai Math., 59 (2014), $41-55$.

[9] A. W. Goodman, On uniformly convex functions, Ann. Polon. Math., 56 (1991), 87-92.

[10] F. Rønning, Uniformly convex functions and a corresponding class of starlike functions, Proc. Amer. Math. Soc., 118 (1993), 189-196.

[11] Ma. W. C and D. Minda, Uniformly convex functions, Ann. Polon. Math., 57 (1992), 165-175.

[12] F. Rønning, On starlike functions associated with parabolic regions, Ann. Univ. Mariae Curie-Sklodowska Sect. A, 45 (1991), 117-122.

[13] S. Kanas and A. Wisniowska, Conic regions and k-uniform convexity, J. Comput. Appl. Math., 105 (1999), 327-336.

[14] S. Kanas and H. M. Srivastava, Linear operators associated with $k$-uniformly convex functions, Integral Transforms Spec. Funct., 9 (2000), 121-132.

[15] W. C. Ma nad D. Minda, A unified treatment of some special classes of univalent functions, in: Proceedings of the Conference on Complex Analysis, Tianjin,1992, 157-169, Conf. Proc. Lecture Notes Anal. I, Int. Press, Cambridge, MA, 1994.

[16] Shuhai Li, Huo Tang, Lina Ma and En Ao, A new class of harmonic multivalent meromorphic functions, Bull. Math. Anal. Appl., 7 (2015), 20-30.

[17] R. M. El-Ashwah and B. A. Frasin, Hadamard product of certain harmonic univalent meromorphic functions, Theory and Applications of Mathematics Computer Science, 5 (2015), 126-131.

[18] H. Silverman, Partial sums of starlike and convex functions, J. Math. Anal. Appl., 209 (1997), 221-227.

[19] E. M. Silvia, On partial sums of convex functions of order $\alpha$, Houston J. Math., 11 (1985), 397-404.

[20] S. Porwal, Partial sums of certain harmonic univalent function, Lobachevskii J. Math., 32 (2011), 366-375.

[21] S. Porwal and K. K. Dixit, Partial sums of starlike harmonic univalent function, Kunpook Math. J., 50 (2010), 433-445.

[22] S. Porwal, A convolution approach on partial sums of certain harmonic univalent function, Internat. J. Math. Math. Sci., 2012, Art. ID 509349, 1-12.

School of Mathematics and Statistics, Chifeng University, Inner Mongolia 024000, P. R. China.

E-mail: lishms66@163.com

E-mail: thth2009@163.com

E-mail: malina00@163.com

E-mail: cfxyaoen@sina.com 